\title{
Employment Consequences of COVID-19 for People with Disabilities and Employers
}

\author{
Jasin Wong ${ }^{1}$ (D) Nnaemezie Ezeife ${ }^{2} \cdot$ Angelika Kudla $^{2} \cdot$ Deborah Crown $^{2} \cdot$ Robert Trierweiler $^{2} \cdot$ Pamela Capraro $^{2}$. \\ Stephanie Tomazin ${ }^{2} \cdot$ Han Su$^{3} \cdot$ Tri Pham $^{4} \cdot$ Allen W. Heinemann ${ }^{2,5}$
}

Accepted: 3 November 2021 / Published online: 17 January 2022

(C) The Author(s), under exclusive licence to Springer Science+Business Media, LLC, part of Springer Nature 2021

\begin{abstract}
Purpose The COVID-19 pandemic has disproportionately affected the lives of people with disabilities (PWD). How the pandemic affects the employment of PWD and employers has yet to be determined. We aimed to investigate the employment consequences of the pandemic as experienced by PWD and employers. The research questions were: (1) What employment effects do PWD experience, and what business changes do employers encounter as a result of the COVID-19 pandemic? (2) What challenges have PWD encountered during the pandemic?Methods Cross-sectional online surveys of 733 PWD and 67 employers in the Midwestern United States.Results Compared to non-disabled peers, PWD encountered more challenges in employment during the pandemic. We found high percentages of both employers and PWD experiencing employment changes and business shutdown during the pandemic. For PWD whose employment was not affected, $14.6 \%$ of the participants $(n=$ 107) expected a loss of income and worried about the economic uncertainty of the pandemic. Unemployment for PWD is high due to illness or disability, being laid-off or furloughed, business reductions, and not feeling safe to work. However, only about $18.6 \%$ of unemployed PWD $(n=16)$ received pay or benefits for the time they were not working even though more than half filed for unemployment benefits.Conclusions The pandemic adversely affected employment of PWD as reported by workers and employers. Findings parallel the experience of the non-disabled workforce, but reveal vulnerabilities that reflect disability consequences and the need for job accommodations. Results reveal emergent needs for policy supports to reduce the disparities experienced by PWD in the workplace.
\end{abstract}

Keywords COVID-19 $\cdot$ Disabled persons $\cdot$ Employment $\cdot$ Return to work

Jasin Wong

jswong@bu.edu

1 Department of Special Education, National Tsing Hua University, No. 521, Nanda Rd. East Dist., Hsinchu, Taiwan

2 Center for Rehabilitation Outcomes Research, Shirley Ryan AbilityLab, 355 East Erie St., Chicago, IL 60611, USA

3 Center for Health Services and Outcomes Research, Feinberg School of Medicine at Northwestern University, 633 N. St. Clair St., Suite 2000, Chicago, IL 60611, USA

4 University of Texas Southwestern Medical School, 5323 Harry Hines Blvd, Dallas, TX 75390, USA

5 Department of Physical Medicine \& Rehabilitation, Feinberg School of Medicine at Northwestern University, 710 N. Lake Shore Dr., Chicago, IL 60611, USA

\section{Introduction}

Coronavirus disease (COVID-19) continues to spread at unprecedented rates. The World Health Organization (WHO) reported over 240 million cases and nearly five million deaths globally as of the end of October 2021 [1]. In the United States (US), there are more than 45.5 million confirmed cases with more than seven hundred thousand deaths [2]. The COVID-19 pandemic also led to the highest unemployment rate since 1948 in the US due to mandatory shutdowns and voluntary business closures [3]. While the emergence of the pandemic and the economic recession affected all demographic groups, it placed significant burden on minority populations, including Blacks, Hispanics, and younger workers as reported in May 2021 by the US Congressional Research Service[4]. In April 2021, 9.4 million persons reported that they are unable to work because their employer closed or lost business due to the pandemic 
[5]. The COVID-19 pandemic greatly affected small businesses, which are responsible for employing half of American workers; $43 \%$ of small businesses temporarily closed, and employment rates decreased by $40 \%$ [6].

As with other population groups, the effects of the COVID-19 pandemic on people with disabilities (PWD) has yet to be described completely [7, 8]. Regarding employment, there were 10 million fewer jobs in November 2020 than in February of that year [5], reflecting a significant increase in layoffs and unemployment for people with and without disabilities [9]. By the end of 2020, the unemployment rate for PWD increased to $12.6 \%$ from a low of $7.8 \%$ at the beginning of the year $[5,10]$. Across age groups, PWD were much more likely to be unemployed compared to their non-disabled peers $[5,11]$. Beyond employment status, PWD reported higher levels of social isolation, disruption in daily activities, and worse quality of life during the pandemic [12-14] than people without disabilities.

Furthermore, workers with disabilities are more likely to be unstably employed and consequently do not receive employment benefits and protections. For example, Emerson, Hatton [15] reported that people with intellectual disabilities were more likely to be exposed to non-standard employment conditions, including temporary contracted employment and part-time jobs, and experience job insecurity and economic inactivity than the general population. The authors also found that unstable employment and job insecurity were associated with poorer health conditions. Delman, Kovich [16] stressed that people with mental illness often experienced challenges accessing reasonable job accommodations and were not eligible for health insurance benefits. The employment picture for PWD was worse than their non-disabled peers during the economic regression from December 2007 to June 2010. McAlpine and Alang [17] reported that people with mental illness and physical disabilities experienced the biggest hits in employment during the Great Recession. PWD were more likely to be unemployed, experience poor living conditions, and use social welfare than their peers without disabilities [17]. Needed are investigations of the employment and economic consequences of the COVID-19 pandemic on PWD.

During the pandemic, PWD encountered increased difficulties maintaining employment due to difficulties managing their daily activities. Even when they have secure employment, they have a higher risk of COVID-19 infection, especially since the pandemic exacerbated barriers to health care $[8,18]$. For example, some PWD were unable to wear masks to protect themselves due to their disabilities or medical conditions. Telehealth access may also be unsuitable for people with sensory impairments or cognitive disabilities [18]. PWD often rely on public and adapted transportation for employment and healthcare, and frequently depend on regular contact from caregivers to accomplish their activities of daily living [8]. Reduced access to healthcare and community support services has a direct effect on PWDs' willingness and ability to acquire and maintain employment.

The risk of discrimination and violation of rights to equitable treatment may further limit the likelihood of employment for PWD [19-22]. During the first three months of the pandemic lockdown, PWD were more likely to experience reduced work hours and financial problems than their nondisabled peers [23]. Workplace disparities reflecting lower job security, may account for the higher unemployment rate for PWD [24]. Scientific publications and advocacy associations are calling for actions to refine the health and work policies, planning, and practice to limit the adverse influences of the pandemic on PWD [25-27].

Although there is preliminary evidence attesting to the effects of COVID-19 on PWD in their daily lives and access to healthcare, there is limited literature investigating how COVID-19 affects PWD's opportunities to obtain and maintain employment. Additionally, there is no research exploring if employers experienced changes during the pandemic that would influence their ability and willingness to support employees with disabilities. Thus, we aimed to investigate the effects of COVID-19 on PWD's employment and understand employers' experience during the pandemic. Two research questions guided this study:

(1) What employment effects do PWD experience, and what business changes do employers encounter as a result of the COVID-19 pandemic?

(2) What challenges have PWD encountered during the pandemic?

\section{Methods}

\section{Research and Survey Design}

We used a cross-sectional design and developed two online surveys to acquire information from PWD and employers who hired PWD, respectively. We aimed to recruit PWD and employers in the Midwest United States, primarily within the state of Illinois. The research was approved by the Institutional Review Board at Northwestern University (STU00208453). The surveys were built and distributed through REDCap [28, 29], a secure online platform for developing and managing surveys. One of the resources used to develop the PWD and employer surveys was a questionnaire developed by the Office of Disability Employment Policy (ODEP) [10]; we adopted and revised some of the COVID-specific questions from the Pandemic Impacts Inventory developed by Grasso, Briggs-Gowan [30]. We 
pilot tested the surveys with 40 PWD and 20 employers and made revisions.

\section{Sample Recruitment}

\section{PWD Survey}

We distributed the PWD survey from October 2020 to March 2021. The eligibility criteria were: (1) at least 18 years old, (2) ability to speak and understand English, and (3) a physical disability. We sent recruitment letters and emails to clients who received vocational rehabilitation services from the Shirley Ryan AbilityLab and from persons who met eligibility criteria using electronic medical records at Shirley Ryan AbilityLab. We sought a broader respondent pool through social media, newsletters, an invitation on the Shirley Ryan AbilityLab patient portal, and consumer organizations. We contacted eligible individuals $2-5$ times to increase the response rate.

Respondents completed an online survey built in REDCap after reviewing instructions, providing consent, and answering eligibility questions. The instructions defined 'physical disability' as a limitation of a person's physical functioning, mobility, dexterity or stamina, which may limit activities of daily living. To ensure potential participants were eligible, they answered three questions before starting the survey: (1) Are you 18 years of age or older? (2) Do you speak English? (3) Do you or did you have a physical disability that affects your ability to work? We added a fourth eligibility question, "Have you ever had a brain injury (including a stroke or brain tumor), a neurological disorder (such as Parkinson's disease) or multiple sclerosis?" to include participants who believed that their neurological injuries or disorders were not a physical disability. People who did not fulfill the eligibility criteria were thanked for their interest and excluded from the survey. Participants selected a primary disability from a list, contained in Online Appendix 1.

\section{Employer Survey}

We recruited participants for the employer survey from August 2020 to March 2021. Eligibility criteria were: (1) age 18 years and older, (2) ability to speak and understand English, and (3) involvement in hiring, accommodations, or managing employees. Respondents viewed instructions, provided consent, then answered eligibility questions on a REDCap platform. We mailed recruitment letters to employers working with our Vocational Rehabilitation Department and their contacts including the Illinois Office of Rehabilitation Services, Disability:IN, the Chicago Mayor's Office for People with Disabilities, and the Great Lakes ADA center. We contacted employers at least three times via email, phone, or mail to enhance the response rate.

\section{Data Analysis}

We used the IBM Statistical Package for the Social Sciences (SPSS) (Version 25.0) for Windows [31] to conduct the statistical analyses. We computed frequencies and percentages to present the descriptive characteristics of the sample. We compared the demographic characteristics of PWD who were versus were not affected by COVID-19 with $t$-tests, chi-square tests, and Fisher's exact test, as appropriate. All statistical tests were two-sided with statistical significance set at 0.05 .

\section{Results}

\section{PWD Survey}

Table 1 displays the demographic information for the PWD sample. A total of 733 PWD were eligible; $54.9 \%$ $(n=346)$ were female. The majority of the participants were White $(72.3 \% ; n=449)$, non-Hispanic $(89.4 \% ; n=$ $554)$, and had a bachelor's or higher degree $(61.2 \%, n=$ 381). Approximately half of the participants were married or living with significant others $(53.1 \% ; n=331)$. The average age was 49 years. The sample was similar to the US population of people with disabilities in terms of gender (female-National: $52.1 \%$ vs. the current sample: $54.9 \%$ ), race (Black-National: $13.7 \%$ vs. $16.0 \%$ ), and ethnicity (Hispanic-National: $13.4 \%$ vs. $10.6 \%$ ) [32, 33]. However, the sample reported greater educational attainment than the US disability population (bachelor's degree or higher-National: $15.8 \%$ vs. $60.5 \%$ ) [32].

About one-quarter $(24.8 \%, n=182)$ of the participants reported that the COVID-19 pandemic affected their employment. Among these respondents, $52.5 \%(n=95)$ were working when they completed the survey, and $47.5 \%$ $(n=86)$ did not work during the pandemic. Employment was related to age $(\mathrm{t}=3.78, p<.001)$, Hispanic ethnicity $\left(\chi^{2}=15.98, p<.001\right)$, and marital status $\left(\chi^{2}=18.51, p\right.$ $<.001)$ such that those who reported pandemic effects tended to be younger, Hispanic, and single. Loss of income within a month was anticipated by $14.6 \%(n=107)$ of the respondents whose employment was not affected yet due to the pandemic and worried about the uncertainty of the economic recession.

Table 2 shows that among PWD whose employment changed since the pandemic, $78.5 \%(n=142)$ reported these changes were related directly to the COVID-19 pandemic. Almost half of the PWD shared that their pay decreased or they had serious financial problems (42.2\%), needed to work remotely or from home more than usual (41.1\%), and were furloughed, on medical leave, or worked reduced hours 
Table 1 Demographic characteristics of people with disabilities who have versus have not been affected by the COVID-19 Pandemic

\begin{tabular}{|c|c|c|c|}
\hline Demographic categories & $\begin{array}{l}\text { PWDs affected } \\
\text { by COVID-19 } \\
(\mathrm{n}=168)\end{array}$ & $\begin{array}{l}\text { PWDs not affected by } \\
\text { COVID-19 }(\mathrm{n}=462)\end{array}$ & Statistical test \\
\hline Gender & & & Fisher's exact $=.55$ \\
\hline Male & $74(44.0 \%)$ & $198(42.9 \%)$ & \\
\hline Female & $90(53.6 \%)$ & $256(55.4 \%)$ & \\
\hline Other & $4(2.4 \%)$ & $8(1.7 \%)$ & \\
\hline Race & & & Fisher's exact $=9.35^{*}$ \\
\hline White/Caucasian & $116(70.7 \%)$ & $333(72.9 \%)$ & \\
\hline Black/African American & $22(13.4 \%)$ & $79(17.3 \%)$ & \\
\hline Asian/Pacific Islander & $4(2.4 \%)$ & $17(3.7 \%)$ & \\
\hline Multiracial & $6(3.7 \%)$ & $9(2.0 \%)$ & \\
\hline Other & $16(9.8 \%)$ & $19(4.2 \%)$ & \\
\hline Ethnicity & & & $\chi^{2}=15.98 * * *$ \\
\hline Hispanic/Latino & $31(18.5 \%)$ & $35(7.6 \%)$ & \\
\hline Age (mean \& standard deviation) & $45.7(14.6)$ & $50.7(14.8)$ & $\mathrm{t}=3.78 * * *$ \\
\hline Education & & & $\chi^{2}=1.34$ \\
\hline $\begin{array}{l}\text { Trade license/Certificate/ High } \\
\text { school diploma/GED or Below }\end{array}$ & $27(16.2 \%)$ & $65(14.3 \%)$ & \\
\hline Associate degree or college & $44(26.3 \%)$ & $106(23.2 \%)$ & \\
\hline Bachelor's degree & $50(29.9 \%)$ & $152(33.3 \%)$ & \\
\hline Doctorate or master's degree & $46(27.5 \%)$ & $133(29.2 \%)$ & \\
\hline Marital status & & & $\chi^{2}=18.51 * * *$ \\
\hline Married & $58(34.7 \%)$ & $240(52.6 \%)$ & \\
\hline Single & $75(44.9 \%)$ & $134(29.4 \%)$ & \\
\hline Divorced/separated/widowed & $22(13.2 \%)$ & $61(13.4 \%)$ & \\
\hline Living with significant other/partner & $12(7.2 \%)$ & $21(4.6 \%)$ & \\
\hline
\end{tabular}

$(40.0 \%)$. More than one fifth of the sample reported that they had a harder time getting their work done (22.8\%) and had increased workload or more work hours (22.2\%) during the pandemic.

For PWD who were not working during the pandemic, most lost their jobs (33.7\%), were on leave (17.4\%), left their jobs (10.5\%), or were temporarily laid off $(9.3 \%)$. The main reasons for not working were: (1) the pandemic-related reduction in business $(47.0 \%)$, (2) being sick or disabled $(31.3 \%)$, and (3) not wanting to work during the pandemic $(22.9 \%)$. Although more than half of the respondents filed for unemployment benefits $(54.4 \%)$, only $18.6 \%$ of the participants received pay or benefits for the time they were not working.

For PWD who were working during the pandemic, $45.3 \%$ were still at the same job; however, there were substantial percentages who found different employment $(27.4 \%)$ or altered their employment model (became self-employed; $20 \%$ ). Despite being able to maintain employment in the pandemic, $67.0 \%$ reported their jobs were moderately or largely affected by the COVID-19.

Finally, we examined relationships between demographic characteristics and employment using $X^{2}$ and Fisher's exact tests; and combined categories for variables with less than five individuals in any subgroup. Married people with disabilities were less likely to (1) lose employment during the pandemic $\left(X^{2}=17.04, p<.001\right)$, (2) encounter difficulty in maintaining work schedule $\left(X^{2}=4.66, p<.05\right)$, (3) experience decreased work pay or serious financial problems $\left(X^{2}=8.13, p<.01\right)$, and (4) have difficulty commuting to work $\left(X^{2}=5.16, p<.05\right)$. Moreover, Hispanic PWD were more likely to experience decreased pay or have serious financial problems $\left(X^{2}=6.06, p<.05\right)$. Pandemic effects on employment were not related to respondents' sex, race, or education level.

\section{Employer Survey}

Table 3 summarizes results from the employer survey; 67 employers or supervisors answered the survey from diverse industries, including health care and social services $(34.0 \%$, $n=18)$, education $(9.4 \%, n=5)$, and arts, entertainment and recreation $(7.5 \%, n=4)$. The majority of the employers represented medium or large businesses with more than 50 employees $(73.6 \%, n=39)$ and worked in the Midwest $(84.9 \%, n=45)$. Most of the respondents worked in human 
Table 2 PWD survey results
Survey questions

Frequency $(\%)$

Have you experienced employment changes since the pandemic?

Yes

$182(24.8 \%)$

No

$551(75.2 \%)$

Only for people whose employment changed during COVID-19

Are you currently working?

Yes

No

$86(47.5 \%)$

How has the COVID-19 outbreak affected you?

Decreased work pay or had serious financial problems

$76(42.2 \%)$

Worked remotely or from home more than you usually do

$74(41.1 \%)$

Furloughed, on the medical leave, or worked reduced hours

$72(40.0 \%)$

Had a harder time getting the work done

$41(22.8 \%)$

Had increased workload, or more work hours

$40(22.2 \%)$

Was not able to maintain regular work schedule

$31(17.2 \%)$

Had difficulty commuting to worksite or arranging job-related travel

$28(15.6 \%)$

Spent a lot of time disinfecting at home

$23(12.8 \%)$

Continued to work even though in close contact with people who might be infected by COVID-19

Was not able to access job accommodations or work-related materials or equipment

Had difficulty communicating with co-workers, employers, or PWDs

Provided care to people with COVID-19

Had difficulty arranging for childcare or need to pay more for childcare expenses

Only for PWD who were NOT currently working $(n=86)$

Which statement best reflects your current employment status?

I lost my job

$29(33.7 \%)$

I am on sick leave or other leave from the same job

$15(17.4 \%)$

I left my job

$9(10.5 \%)$

I have been temporarily laid off from the same job

$8(9.3 \%)$

I stopped my job search because few employers are hiring

$5(5.8 \%)$

I closed my business temporarily

$3(3.5 \%)$

What is your main reason for not working for pay or profit?

I did not work due to pandemic-related reduction in business

$39(47.0 \%)$

I am/was sick or disabled

$26(31.3 \%)$

I was not able to find a job or did not want to work at this time

$19(22.9 \%)$

I did not work because I am or was caring for someone

$7(8.4 \%)$

I am retired

$7(8.4 \%)$

Have you filed for unemployment benefits?

Yes

$31(54.4 \%)$

No

$26(45.6 \%)$

Are you receiving pay for the time you are not working?

Yes (paid leave fully and partially)

$16(18.6 \%)$

No

$70(81.4 \%)$

Only for PWD who were currently working $(n=95)$

Which statement best reflects your current employment status?

I am still at my same job

I found new or different employment

I altered the way business was conducted or changed the business model

$17(17.9 \%)$

I changed my business model

$2(2.1 \%)$

To what extent has COVID-19 affected your work?

My work is largely affected by COVID-19

$32(34.0 \%)$

My work is moderately affected by COVID-19 
Table 2 (continued)

\begin{tabular}{lc}
\hline Survey questions & Frequency (\%) \\
\hline My work is somewhat affected by COVID-19 & $25(26.6 \%)$ \\
COVID-19 has not affected my work & $6(6.4 \%)$ \\
\hline
\end{tabular}

Table 3 Employer characteristics

\begin{tabular}{lr}
\hline Company size & \\
\hline $1-14$ & $6(11.3 \%)$ \\
$15-49$ & $7(13.2 \%)$ \\
$50-249$ & $15(28.3 \%)$ \\
$250-999$ & $8(15.1 \%)$ \\
1000 or more & $16(30.2 \%)$ \\
Company location & \\
Midwest & $46(86.8 \%)$ \\
Others & $7(13.3 \%)$ \\
Business headquarter location & \\
Midwest & $45(84.9 \%)$ \\
Others & $8(15.2 \%)$ \\
Job title of the responder & \\
Health science & $22(42.3 \%)$ \\
Human services & $12(23.1 \%)$ \\
Business management and administration & $5(9.6 \%)$ \\
Job tenure of the responder (years) & \\
$0-4$ & $26(49.1 \%)$ \\
$5-9$ & $12(22.6 \%)$ \\
$10-19$ & $6(11.3 \%)$ \\
$20-29$ & $6(11.3 \%)$ \\
$30+$ & $3(5.7 \%)$ \\
\hline
\end{tabular}

services and health science industries; job tenure was 0-4 years for $49.1 \%(n=26)$. Compared to the 2015 Harris PollNielsen National Survey [34], our respondents were less likely to work for middle and small businesses (company size 1-999 employees-National: $83.5 \%$ vs. $67.9 \%$ ) and more likely to work for large companies (1000+ employeesNational: $16.5 \%$ vs. $30.2 \%$ ). Respondents were also more likely to work in Healthcare and Social Services (Midwest: $19.2 \%$ vs. $34.0 \%$ ), while other industry categories were underrepresented, such as manufacturing (Midwest: $14.9 \%$ vs. $3.8 \%$ ) and retail (Midwest: $11.4 \%$ vs. 1.9\%) [35].

Table 4 presents the COVID-19 pandemic effects on respondents' businesses. All but 2 of the respondents $(97.0 \%, n=65)$ reported that their business was affected by COVID-19. Major effects included changing to virtual or remote operations $(68.8 \%)$, altering how they conduct business $(62.5 \%)$, furloughing or laying-off employees $(47.5 \%)$, and temporarily closing (26.3\%). Of the employers who closed or changed their business, $66.3 \%$ were concerned about the spread of COVID-19, $45.0 \%$ stated that government policies required them to close, and $28.7 \%$ had difficulties providing services during the pandemic.
Table 4 Employer survey results

\begin{tabular}{lc}
\hline Survey questions & Frequency (\%) \\
\hline Has COVID-19 impacted your business? & \\
Yes & $65(97.0 \%)$ \\
No & $2(3.0 \%)$ \\
How has COVID-19 impacted your business? & $55(68.8 \%)$ \\
Changed to virtual/remote operations & $50(62.5 \%)$ \\
Altered the way business was conducted & $21(26.3 \%)$ \\
Closed the business/organization temporarily & $20(25.0 \%)$ \\
Furloughed employees & $18(22.5 \%)$ \\
Laid-off employees & \\
Reasons for closing or changing the business & $53(66.3 \%)$ \\
We worry about the spread of COVID-19 & $36(45 \%)$ \\
The government policies required us to close & $23(28.7 \%)$ \\
Difficult to provide services during the pandemic & $14(17.5 \%)$ \\
Loss customers during the COVID-19 pandemic & $10(12.5 \%)$ \\
Business Partners required us to close or change our business & $7(8.8 \%)$ \\
The operating cost is too high to manage & $5(6.3 \%)$ \\
Difficult to find marketing channels or delivery methods & $5(6.3 \%)$ \\
We cannot find enough employees & $3(3.8 \%)$ \\
Increased challenges in obtaining supplies &
\end{tabular}




\section{Discussion}

This study provides important information about how the COVID-19 pandemic affects the employment status of PWD and businesses of employers mainly in the Midwestern United States. PWD encountered significant challenges obtaining or maintaining employment in a safe workplace during the pandemic-related economic recession. Overall, $97.0 \%$ and $24.8 \%$ of employers and PWD experienced employment changes and business shutdowns during the pandemic, respectively. For PWD whose employment was not affected, $14.6 \%$ expected a loss of income and were worried about their financial security. Nearly half $(47.5 \%)$ of PWD were unemployed due to illness or disability, being laid-off, or not feeling safe to work. However, only $18.6 \%$ of unemployed PWD received pay or benefits for the time they are not working even though more than half filed for unemployment benefits. Perhaps PWD were ineligible for benefits because they had shorter or interrupted work histories. If so, government policies could reduce this disparity in unemployment benefits by considering the unique needs of PWD that limit full time employment.

Some PWD are at a high risk of infection from COVID19 due to compromised immune and respiratory systems. Social distancing caused disruptions in care for PWD who rely on home health or personal assistants [21]. Despite the significant effects of the pandemic on PWD, the Executive and Legislative branches of the federal government made no disability-specific policy changes. Labor policies established by the US Department of Labor and monitored by the National Conference of State Legislatures, 2020, such as the Family First Coronavirus Response Act and the Coronavirus Aid, Relief, and Economic Security (CARES) program, offered financial support to Americans unable to work due to the pandemic [36] regardless of disability status. As people are getting vaccinated in the US and businesses are re-opening, employers will need to adhere to the Equal Employment Opportunity Commission (EEOC) guidelines regarding the Americans with Disabilities Act [37]. Well-designed and sustained labor along with public health policies were critical during the pandemic. Policies should also address PWD's needs for healthcare services, employment, transportation, smart technologies, and communication technology.

Employers and human resource professionals should remain vigilant about job accommodations for PWD and cognizant of additional needs that may arise when creating a reopening plan. Difficulties may emerge from both direct health risks related to COVID-19 as well as work protocols enacted in response to the pandemic [8]. In the latter cases, employers should consider providing work space modifications to allow PWD to meet hygiene protocols, such as Americans with Disabilities Act (ADA)-compliant bathrooms, placement of motion-activated sanitizer dispensers within reach of wheelchair users, and storing disinfectant wipes on lower shelves [38]. For employees with cognitive or visual deficits, employers should provide reminders of protocols in relevant spaces (e.g., large poster above a sink for effective handwashing techniques) and mark boundaries for social distancing [38]. They should make special efforts to support employees who are deaf or hard-of-hearing in virtual work environments, including using quiet rooms, avoiding background noises, creating protocols to avoid crosschatter, and implementing visual text [39]. Companies may implement adaptive technologies and provide opportunities to bring home work equipment and software [39]. Finally, employers may also collaborate with PWD to create accessible screening questionnaires for COVID-19 symptoms and allow an option for remote completion.

It is imperative that employers offer appropriate physical protection to individuals whose disability puts them at increased risk for infection. At a minimum, employers should provide options for one-way aisles, Plexiglas barriers, non-latex gloves, gowns designed for wheelchair-users, and modified face masks with clear center panels for lipreaders and interpreters [38, 40]. The ADA also maintains that receiving accommodations does not preclude PWD from requesting additional protections for COVID-19-related concerns. In summary, it is critical to remain flexible and responsive to the needs of employees with disabilities, understanding that they may change with the emerging practices and guidelines from the Centers for Disease Control and Prevention.

The delivery of rehabilitation services, including vocational rehabilitation, was altered by the pandemic [41]. Inperson services were postponed or restricted and providers developed new ways of delivering services which included the rapid expansion of telehealth. Along with telehealth, working remotely has increased the need to assist employees with disabilities in determining, accessing, and implementing reasonable accommodations in their home environments [40]. Vocational rehabilitation counselors and occupational therapists are well positioned to work with employers and employees with disabilities in ensuring the at-home work environment accommodates employees. The need to support COVID-19 long haulers return to work is another role for rehabilitation counselors given their knowledge of the ADA [42, 43]. Since we are still learning about the longterm effects of the COVID-19 pandemic on PWD [7, 8], the scope of new opportunities for rehabilitation professionals is just being realized.

Our study has several strengths, including assessing whether and how COVID-19 affects PWD and their employers' work status and businesses. We consulted with an advisory group composed of key stakeholders and conducted 
cognitive interviews with participants to validate the surveys before dissemination. However, this study has some limitations. First, the cross-sectional design does not capture the influences of COVID-19 on PWD's employment over time as the social, economic, and political situations changed rapidly during the pandemic. Longitudinal studies should investigate the long-term effects of the pandemic on PWD's employment. Second, inherent in the sampling approach, our participants were restricted to PWD with internet access, understood English, and lived primarily in the Midwest. This sampling strategy could limit the generalizability of the results. Future studies should explore regional and industry variations nationwide and translate the surveys into different languages to obtain perspectives from diverse populations.

In summary, a majority of employers experienced adverse effects due to the COVID-19 pandemic, and a quarter of PWD experienced effects resulting from the COVID-19 pandemic, most significantly by PWD who are younger, single, and Hispanic. These insights into the pandemic from the perspective of employers and PWD underscore pervasive disparities in healthcare and employment. Future research should evaluate these disparities across industries with a particular focus on jobs that cannot be performed remotely.

Supplementary Information The online version contains supplementary material available at https://doi.org/10.1007/s10926-021-10012-9.

Acknowledgements We appreciate the efforts of the participants who shared their work experience during the pandemic. This research was supported by the National Institute on Disability, Independent Living, and Rehabilitation Research (NIDILRR) (Grant Number: 90RTEM0001).

Author Contributions AWH is the project director. DC, RT, PC, AWH, $\mathrm{JW}$, and AK participated in survey development. DC, RT, PC, AK, NE, ST were involved in data collection. JW, NE, AK, and ST managed and analyzed the data. All authors participated in results interpretation, and manuscript writing, reviewing and editing. All authors approved the manuscript to be submitted and published.

Funding This study was funded by the National Institute on Disability, Independent Living, and Rehabilitation Research (NIDILRR) (Grant Number: 90RTEM0001)

Data Availability The datasets generated during and analyzed during the current study are not publicly available.

Code Availability Not applicable.

\section{Declarations}

Conflict of interest The authors have no conflicts of interest to declare that are relevant to the content of this article.

Ethical Approval This study was reviewed and approved by the Institutional Review Board at Northwestern University (STU00208453).
Consent to Participate All procedures followed were in accordance with the ethical standards of the responsible committee on human experimentation (institutional and national) and with the Helsinki Declaration of 1975, as revised in 2000 (5). Online informed consent was obtained from all individual participants for being included in the study.

Consent for Publication We confirm that this work is original and has not been published elsewhere, nor is it currently under consideration for publication elsewhere. We consent to publish the results through Journal of Occupational Rehabilitation.

\section{References}

1. World Health Organization. WHO coronavirus disease (COVID-19) Dashboard. 2021 https://covid19.who.int/?gclid= CjwKC Ajww5 r8BRB 6EiwA rcckC53o8XgWLdGgg4gH JJJY_tu1yc3vfDxb_RmgDaYmfy3Out2shs7LJhoC0FIQAvD_ BwE. Accessed 16 Feb 2021

2. John Hopkins University. COVID-19 data in motion. 2021 https:// coronavirus.jhu.edu/. Accessed 3 Jun 2021

3. National Conference of State Legislatures. COVID-19: impact on employment and labor. 2020. https://www.ncsl.org/research/ labor-and-employment/covid-19-impact-on-employment-andlabor.aspx. Accessed 3 Jun 2021

4. Falk, G., et al. Unemployment rates during theCOVID-19 pandemic. 2021 https://crsreports.congress.gov/product/pdf/R/ R46554/13. Accessed 20 Jul 2021

5. Bureau of Labor Statistics. Persons with a disability: labor force characteristics - 2020. 2021 https://www.bls.gov/news.release/ pdf/disabl.pdf. Accessed 6 Jul 2021

6. Bartik, A.W., et al., The impact of COVID-19 on small business outcomes and expectations. Proc Natl Acad Sci USA, 2020. 117(30): p. 17656-17666.

7. Bernard, A., et al., Assessing the impact of COVID-19 on persons with disabilities: development of a novel survey. Int J Public Health, 2020. 65(6): p. 755-757.

8. Lebrasseur A, et al. Impact of COVID-19 on people with physical disabilities: a rapid review. Disabil Health J. 2021;14(1):101014.

9. Houtenville AJ, Paul S, Brucker DL. Changes in the employment status of people with and without disabilities in the United States during the COVID-19 pandemic. Arch Phys Med Rehabil. 2021;102(7):1420-1423.

10. Office of Disability Employment Policy. Employment for persons with a disability: analysis of trends during the COVID-19 pandemic 2020. https://www.dol.gov/agencies/oasp/evaluation/ completedstudies/Employment-for-Persons-with-Disability-Analy sis-of-Trends-During-COVID-19-Pandemic. Accessed 6 Jul 2021

11. Kaye HS. The impact of the 2007-09 recession on workers with disabilities. Monthly Labor Rev. 2010;133(10):19-30.

12. American Psychological Association. How COVID-19 impacts people with disabilities 2020. https://www.apa.org/topics/covid19/research-disabilities. Accessed 24 May 2021

13. Epstein $\mathrm{S}$, et al. New obstacles and widening gaps: a qualitative study of the effects of the COVID-19 pandemic on U.S. adults with disabilities. Disabil Health J. 2021. https://doi.org/10.1016/j. dhjo.2021.101103.

14. Steptoe, A. and G. Di Gessa, Mental health and social interactions of older people with physical disabilities in England during the COVID-19 pandemic: a longitudinal cohort study. Lancet Public Health, 2021. 6(6): p. e365-e373. 
15. Emerson E, et al. The association between non-standard employment, job insecurity and health among British adults with and without intellectual impairments: cohort study. SSM Popul Health. 2018;4:197-205.

16. Delman, J., et al., The promise of demand side employer-based strategies to increase employment rates for people living with serious mental illnesses. Psychiatr Rehabil J, 2017. 40(2): p. 179-182.

17. McAlpine DD, Alang SM. Employment and economic outcomes of persons with mental illness and disability: the impact of the great recession in the United States. Psychiatr Rehabil J. 2021;44(2):132-41.

18. Amirhosseini, Z. COVID-19's impact on people with disabilities. 2020. https://www.massgeneral.org/news/coronavirus/Covid-19simpact-on-people-with-disabilities. Accessed 17 Dec 2021

19. Rotarou, E.S., et al., Disabled people in the time of COVID-19: identifying needs, promoting inclusivity. J Glob Health, 2021. 11: p. 03007.

20. Lund, E.M. and K.B. Ayers, Raising awareness of disabled lives and health care rationing during the COVID-19 pandemic. Psychol Trauma, 2020. 12(S1): p. S210-S211.

21. Pineda VS, Corburn J. Disability, urban health equity, and the coronavirus pandemic: promoting cities for all. J Urban Health. 2020;97(3):336-341.

22. Kendall $\mathrm{E}$, et al. Immediate and long-term implications of the COVID-19 pandemic for people with disabilities. Am J Public Health. 2020;110(12):1774-1779.

23. Emerson E, et al. The impact of disability on employment and financial security following the outbreak of the 2020 COVID-19 pandemic in the UK. J Public Health (Oxf). 2021. https://doi.org/ 10.1093/pubmed/fdaa270.

24. Schur L, et al. Disability at work: a look back and forward. J Occup Rehabil. 2017;27(4):482-497.

25. Izutsu, T. Disability-inclusive disaster risk reduction and humanitarian action: an urgent global imperative. United Nations World Conference on Disaster Risk Reduction and the Progress Thereafter 2019 https://www.un.org/development/desa/disabilities/wpcontent/uploads/sites/15/2020/03/Final-Disability-inclusive-disas ter.pdf. Accessed 26 May 2021

26. Allen, P.M. and L. Smith, SARS-CoV-2 self-isolation: recommendations for people with a vision impairment. Eye (Lond), 2020. 34(7): p. 1183-1184.

27. Kessler Foundation. COVID-19 and spinal cord injury: minimizing risks for complications. 2020. https://kesslerfoundation.org/ info/covid-19-and-spinal-cord-injury-minimizing-risks-complicati ons. Accessed 26 May 2021

28. Harris PA, et al. Research electronic data capture (REDCap) - a metadata-driven methodology and workflow process for providing translational research informatics support. J Biomed Inform. 2009;42(2):377-381.

29. Harris, P.A., et al., The REDCap consortium: Building an international community of software platform partners. J Biomed Inform, 2019. 95: p. 103208

30. Grasso, D., et al., A person-centered approach to profilingCOVIDrelated experiences in the united states: preliminary findings from the epidemic-pandemic impacts inventory (EPII). PsyArXiv, 2020.

31. IBM SPSS Statistics for Windows. 2017, IBM Corp.: Armonk, NY.

32. Yang, K.L. and H.E. Tan. Disability statistics: online resource for U.S. disability statistics. 2018. https://www.disabilitystatistics.org/ reports/acs.cfm?statistic $=1$. Accessed 19 Oct 2020

33. Okoro CA, et al. Prevalence of disabilities and health care access by disability status and type among adults-United States, 2016. Morbidity Mortal Wkly Rep. 2018;67(32):882-887.

34. McCleary K, et al. Employer and employee opinions about workplace health promotion (wellness) programs: results of the 2015 Harris Poll Nielsen Survey. J Occup Environ Med. 2017;59(3):256-263.

35. Statistical Atlas. Industries in the Midwest region. 2021. https:// statisticalatlas.com/region/Midwest/Industries. Accessed 24 Oct 2021

36. National Conference of State Legislatures. Labor and employment. 2021. https://www.ncsl.org/research/labor-and-emplo yment/covid-19-impact-on-employment-and-la.aspx. Accessed 10 Jun 2021

37. U.S. Equal Employment Opportunity Commission. What you should know about COVID-19 and the ADA, the rehabilitation act, and other EEO laws. 2020. https://www.eeoc.gov/wysk/whatyou-should-know-about-covid-19-and-ada-rehabilitation-act-andother-eeo-laws. Accessed 29 Mar 2021

38. Center for Inclusive Design and Innovation. Accommodation problems and solutions for reopening with COVID-19. 2021. https://gatfl.gatech.edu/tflwiki/images/8/8d/Return_to_ Work-07.31.2020.pdf. Accessed 17 May 2021

39. Center for Inclusive Design and Innovation. Accommodation problems and solutions associated with telework. 2021. https:// gatfl.gatech.edu/tflwiki/images/d/d7/Telework_Accommodations_ v2.pdf. Accessed 17 May 2021

40. Employer Assistance and Resource Network on Disability Inclusion. COVID-19 and Job applicants and employees with disabilities: emerging practices to employ and protect workers. 2020. https://askearn.org/wp-content/uploads/2020/08/EARN_ 2020_Covid19-PolicyBrief.pdf. Accessed 17 May 2021

41. Jesus TS, Landry MD, Jacobs K. A 'new normal' following COVID-19 and the economic crisis: using systems thinking to identify challenges and opportunities in disability, telework, and rehabilitation. Work. 2020;67(1):37-46.

42. Dill, J.R. Will COVID-19 'long-haulers' be next to test the limits of the ADA? 2020. https://ogletree.com/insights/will-covid-19long-haulers-be-next-to-test-the-limits-of-the-ada/. Accessed 4 Mar 2021

43. Wong J, Kudla A, Pham T, et al. Lessons learned by rehabilitation counselors and physicians in services to COVID-19 long-haulers: a qualitative study. Rehabil Couns Bull. 2021. https://doi.org/10. $1177 / 00343552211060014$

Publisher's Note Springer Nature remains neutral with regard to jurisdictional claims in published maps and institutional affiliations. 